PUPT-1347, IASSNS-HEP-92/60

October 1992

\title{
Static and Dynamic Critical Phenomena at a Second Order QCD Phase Transition
}

\author{
KRISHNA RAJAGOPAL * \\ Department of Physics \\ Joseph Henry Laboratories \\ Princeton University \\ Princeton, N.J. 08544 \\ FRANK WILCZEK $^{\dagger}$ \\ School of Natural Sciences \\ Institute for Advanced Study \\ Olden Lane \\ Princeton, N.J. 08540
}

\footnotetext{
$\star$ Research supported in part by a Natural Sciences and Engineering Research Council of Canada 1967 Fellowship. RAJAGOPAL@PUPGG.PRINCETON.EDU

$\dagger$ Research supported in part by DOE grant DE-FG02-90ER40542. WILCZEK@IASSNS.BITNET
} 


\begin{abstract}
In QCD with two flavors of massless quarks, the chiral phase transition is plausibly in the same universality class as the classical four component Heisenberg antiferromagnet. Therefore, renormalization group techniques developed in the study of phase transitions can be applied to calculate the critical exponents which characterize the scaling behaviour of universal quantities near the critical point. This approach to the QCD phase transition has implications both for lattice gauge theory and for heavy ion collisions. Future lattice simulations with longer correlation lengths will be able to measure the various exponents and the equation of state for the order parameter as a function of temperature and quark mass which we describe. In a heavy ion collision, the consequence of a long correlation length would be large fluctuations in the number ratio of neutral to charged pions. Unfortunately, we show that this phenomenon will not occur if the plasma stays close to equilibrium as it cools. If the transition is far out of equilibrium and can be modelled as a quench, it is possible that large volumes of the plasma with the pion field correlated will develop, with dramatic phenomenological consequences.
\end{abstract}




\section{Introduction}

The QCD phase transition is of interest from several different points of view. First, there can be no doubt that it occurred in the early universe. Second, it is reasonable to hope that in a heavy ion collision of sufficiently high energy, a small region of the high temperature phase is created which then cools through the phase transition. Third, lattice gauge theory is well suited to calculating the equilibrium properties of QCD at high temperatures. From all these perspectives, it is important to learn as much as can be learned analytically about the phase transition, relying as much as possible only on fundamental symmetries and universality arguments and as little as possible on specific assumptions and models.

In a previous paper [1], one of us (F. W.) emphasized that in the chiral limit where there are two species of quarks with zero current algebra mass, the order parameter for the chiral phase transition has the same symmetry as the magnetization of a four component Heisenberg magnet, which has a second order phase transition. In this case, and indeed for any number of quark species except zero, there is no order parameter for a confinement/deconfinement phase transition. Thus in the $m_{u}=m_{d}=0$ limit the universal characteristics of the QCD phase transition (i.e. those characteristics determined by the modes which develop long correlation

lengths at the phase transition) are the same as those of the $N=4$ Heisenberg magnet.

In this paper we further explore the consequences of this approach to the QCD phase transition. In the following section, we review the scenario described in [1], and establish a dictionary between QCD and the magnetic system. In order to make the present paper self-contained, we also include in this section many of the results from [1]. In section 3 , we discuss the behaviour of the pion and sigma masses at the transition. In section 4 , we discuss the $\rho$ and $A_{1}$ mesons. In section 5 , we consider how the strange quark affects the phase transition. In section 6 , we go beyond the static critical phenomena of the earlier sections and discuss the dynamics of the appropriate universality class. In section 7 , we discuss the 
implications of all this for cosmology and lattice gauge theory, and for heavy ion collisions under the assumption that the plasma remains close to thermal equilibrium through the phase transition. In section 8 , we consider what phenomena we can expect in heavy ion collisions if the system gets far out of thermal equilibrium and can be modelled as a quench. Finally, in the last section we summarize and conclude.

\section{QCD and the $O(4)$ Magnet}

As discussed in [1], the physics of the QCD phase transition is qualitatively different in the cases of zero, one, two, or three or more flavors of quarks. In this section we consider QCD with two species of quarks. (An analysis similar to the one which follows leads to the conclusion that for three or more flavors of massless quarks, the chiral phase transition is first order. See [1] for details.) If there are two flavors of massless quarks, the lagrangian is symmetric under global chiral transformations in the group $S U(2)_{L} \times S U(2)_{R} \times U(1)_{L+R}$ of independent special unitary transformations of the left and right handed quark fields, and a vector $U(1)$ transformation which corresponds to baryon number symmetry. (The axial $U(1)$ which would make the symmetry group into $U(2) \times U(2)$ is a symmetry of the classical theory, but not of the quantum theory [2].) This chiral symmetry breaks spontaneously down to $S U(2)_{L+R} \times U(1)_{L+R}$ at low temperatures; and is restored at sufficiently high temperatures. The order parameter for this phase transition is the expectation value of the quark bilinear

$$
\mathcal{M}_{j}^{i} \equiv\left\langle\bar{q}_{L}^{i} q_{R j}\right\rangle
$$

which breaks the symmetry when it acquires a non-zero value below some critical temperature $T_{c}$.

In order to describe a second-order transition quantitatively, we must find a tractable model in the same universality class. For the chiral order parameter (2.1) 
the relevant symmetries are independent unitary transformations of the left- and right-handed quark fields, under which

$$
\mathcal{M} \rightarrow U^{\dagger} \mathcal{M} V
$$

These transformations generate an $U(2)_{L} \times U(2)_{R}$ symmetry, which is not quite what is needed, since it includes the axial baryon number symmetry which is not present in QCD. This problem is solved [1] by restricting $\mathcal{M}$ to unitary matrices with positive determinant, instead of general complex matrices. Matrices $\mathcal{M}$ in this restricted class remain in this restricted class under the transformation (2.2) only if $U$ and $V$ have equal phases. Hence, the axial $U(1)$ has indeed been removed. The $2 \times 2$ matrices $\mathcal{M}$ can conveniently be parametrized using four real parameters $(\sigma, \vec{\pi})$ and the Pauli matrices as

$$
\mathcal{M}=\sigma+i \vec{\pi} \cdot \vec{\tau}
$$

In fact the order parameter can be written as a four-component vector $\phi \equiv(\sigma, \vec{\pi})$ and the transformations (2.2) are simply $O(4)$ rotations in internal space. Hence, the order parameter appropriate for the chiral phase transition in QCD with two flavors of massless quarks has the symmetries of the standard $O(4)$ invariant $N=4$ Heisenberg magnet. For smaller number of components, this sort of model is a much-studied model for the critical behavior of magnets, with the order parameter representing the magnetization of a ferromagnet or the staggered magnetization of an antiferromagnet.

If the phase transition is second order, then it will correspond to an infrared fixed point of the renormalization group. We wish to describe those aspects of the critical behaviour which are universal, that is, those aspects which are determined by the scaling behaviour of operators near the infrared fixed point of the renormalization group. Hence, it is sufficient to retain those degrees of freedom which develop large correlation lengths at the critical point. These are just long 
wavelength fluctuations of the order parameter, which is small in magnitude near the critical point and therefore fluctuates at little cost in energy. Thus the most plausible starting point for analyzing the critical behavior of a second-order phase transition in QCD is the Landau-Ginzburg free energy

$$
F=\int d^{3} x\left\{\frac{1}{2} \partial^{i} \phi^{\alpha} \partial_{i} \phi_{\alpha}+\frac{\mu^{2}}{2} \phi^{\alpha} \phi_{\alpha}+\frac{\lambda}{4}\left(\phi^{\alpha} \phi_{\alpha}\right)^{2}\right\}
$$

Here $\mu^{2}$ is the temperature-dependent renormalized (mass) ${ }^{2}$, which is negative below and positive above the critical point, while $\lambda$ is the strength of the quartic couplings and is supposed to be smooth at the transition. We neglect terms with higher powers of $\phi$ since $|\phi|$ is small near the transition. The symmetry breaking pattern we want is $\mathcal{M} \propto \mathbf{1}$ (equivalently, $\langle\sigma\rangle \neq 0 ;\langle\vec{\pi}\rangle=0$ ) below the transition which is indeed what we find at the minimum of the potential for positive $\lambda$. This model has been studied in depth for arbitrary $N$ and spatial dimension $d$, and the existence of an infrared stable fixed point of the renormalization group has been established [3]. Hence, it is a model for a second order QCD chiral phase transition for two massless quarks.

When the free energy (2.4) is written in terms of $\sigma$ and $\vec{\pi}$ it looks much like the original model of Gell-Mann and Levy [4] with two changes: there are no nucleon fields and only three (spatial) dimensions. These two changes reflect an important distinction [5]. We are only proposing (2.4) as appropriate near the second order phase transition point. This is because it is only there that we can appeal to universality - the long-wavelength behaviour of the $\sigma$ and $\vec{\pi}$ fields is determined by the infrared fixed point of the renormalization group, and microscopic considerations are irrelevant to it. In Euclidean field theory at finite temperature, the integral over $\omega$ of zero temperature field theory is replaced by a sum over Matsubara frequencies $\omega_{n}$ given by $2 n \pi T$ for bosons and $(2 n+1) \pi T$ for fermions with $n$ an integer. Hence, one is left with a Euclidean theory in three spatial dimensions with massless fields from the $n=0$ terms in the boson sums and massive fields from the rest of the boson sums and the fermion sums. Hence, to discuss the massless modes 
of interest at the critical point, (2.4) is sufficient. This means that we do not need to worry whether to introduce nucleon fields as in [4], or constituent quark fields as, for example, in [6].

We have motivated a very definite hypothesis for the nature of the phase transition for QCD with two species of massless quarks, namely that it is in the universality class of the $N=4$ Heisenberg magnet. This hypothesis has numerous consequences, which are the subject of the rest of this paper. To keep the discussion self-contained, in the remainder of this section we review the predictions for the static critical exponents described in [1].

\subsection{CRitical exponents}

First, we define the reduced temperature $t=\left(T-T_{c}\right) / T_{c}$. The exponents

$\alpha, \beta, \gamma, \eta$, and $\nu$ describe the singular behaviour of the theory with strictly zero quark masses as $t \rightarrow 0$. For the specific heat one finds œ[6

$$
C(T) \sim|t|^{-\alpha}+\text { less singular. }
$$

The behaviour of the order parameter defines $\beta$.

$$
\langle|\phi|\rangle \sim|t|^{\beta} \text { for } t<0
$$

$\eta$ and $\nu$ describe the behaviour of the correlation length $\xi$ where

$$
G_{\alpha \beta}(x) \equiv\left\langle\phi(x)_{\alpha} \phi(0)_{\beta}\right\rangle-\left\langle\phi_{\alpha}\right\rangle\left\langle\phi_{\beta}\right\rangle \rightarrow \delta_{\alpha \beta} \frac{A}{|x|} \exp (-|x| / \xi) \text { at large distances. }
$$

$A$ is independent of $|x|$, but may depend on $t$. The correlation length exponent $\nu$ is defined by

$$
\xi \sim|t|^{-\nu}
$$

Above $T_{c}$, where the correlation lengths are equal in the sigma and pion channels, 
the susceptibility exponent $\gamma$ is defined by

$$
\int d^{3} x G_{\alpha \beta}(x) \sim t^{-\gamma}
$$

We will discuss the behaviour of the susceptibility below the transition in the following section. The exponent $\eta$ is defined through the behaviour of the Fourier transform of the correlation function:

$$
G_{\alpha \beta}(k \rightarrow 0) \sim k^{-2+\eta}
$$

The last exponent, $\delta$ is related to the behaviour of the system in a small magnetic field $H$ which explicitly breaks the $O(4)$ symmetry. Let us first show that in a QCD context, $H$ is proportional to a common quark mass $m_{u}=m_{d} \equiv$ $m_{q}$. This common mass term may be represented by a $2 \times 2$ matrix $\mathcal{D}$ given by $m_{q}$ times the identity matrix. We are now allowed to construct the free energy from invariants involving both $\mathcal{D}$ and $\mathcal{M}$. The lowest dimension term linear in $\mathcal{D}$ is just $\operatorname{tr} \mathcal{M}^{\dagger} \mathcal{D}=m_{q} \sigma$, which in magnet language is simply the coupling of the magnetization to an external field $H \propto m_{q}$. In the presence of an external field, the order parameter is not zero at $T_{c}$. In fact,

$$
\langle|\phi|\rangle(t=0, H \rightarrow 0) \sim H^{1 / \delta}
$$

The six critical exponents defined above are related by four scaling relations [3]. These are

$$
\begin{aligned}
\alpha & =2-d \nu \\
\beta & =\frac{\nu}{2}(d-2+\eta) \\
\gamma & =(2-\eta) \nu \\
\delta & =\frac{d+2-\eta}{d-2+\eta}
\end{aligned}
$$

We therefore need values for $\eta$ and $\nu$ for the four component magnet in $d=3$. These were obtained in the remarkable work of Baker, Meiron and Nickel[7], who 
carried the perturbation theory to seven-loop order, and used information about the behaviour of asymptotically large orders, and conformal mapping and Padé approximant techniques to obtain

$$
\begin{aligned}
& \eta=.03 \pm .01 \\
& \nu=.73 \pm .02 .
\end{aligned}
$$

Using (2.12), the remaining exponents are $\alpha=-0.19 \pm .06, \beta=0.38 \pm .01$, $\gamma=1.44 \pm .04$ and $\delta=4.82 \pm .05$. Since $\alpha$ is negative there is a cusp in the specific heat at $T_{c}$, rather than a divergence.

Very different critical exponents are proposed in [8]. These authors thus implicitly claim that a hitherto unknown fixed point theory with the symmetries of the $N=4$ isotropic Heisenberg model exists, and governs the QCD phase transition.

\section{The Equation of State and the Pion and Sigma Masses}

The expressions which define $\beta, \gamma$ and $\delta$ are actually special cases of a more general relationship between the magnetization and the magnetic field called the critical equation of state. The equation of state has been calculated to order $\epsilon^{2}$ by Brézin, Wallace and Wilson [9]. Their result is reproduced in the appendix. In this section, we will use the equation of state to determine the behaviour of the masses of the pion and sigma masses near the critical point.

First, we must define what we mean by the "mass" of the pion and sigma. We could choose either to define the mass as an inverse correlation length or as an inverse susceptibility. We choose the latter, which is conventional in the condensed matter literature. Specifically, we define

$$
m_{\sigma}^{-2}=\int d^{3} x G_{00}
$$

and

$$
m_{\pi}^{-2} \delta_{i j}=\int d^{3} x G_{i j}
$$

where $\phi_{0}=\sigma$ and $\phi_{i}=\pi_{i}, i=1,2,3$. At any given $t$ and $H,(2.7)$ implies that 
whether one defines the mass as the inverse correlation length or as the inverse susceptibility is academic. However, since $A$ in (2.7) depends on $t$ and $H$, the two different choices lead to different scaling behaviours for masses as functions of $t$ and $H$. We shall see that with the conventional choice (3.1) and (3.2), the masses can be extracted conveniently from the equation of state. It is worth noting that the masses we have defined are related only to the behaviour of spatial correlation functions in the static (equilibrium) theory. They carry no dynamical information. Also, we will only be able to make universal statements about how the masses scale at the transition. Normalizing the magnitudes of the masses (i.e. relating them to the zero temperature masses) will require using some specific model, and hence will not be universal.

The equation of state gives the magnetization as a function of $t$ and $H$. For the rest of this paper, we will write the order parameter as $M$, for magnetization, keeping in mind that $M=\langle\sigma\rangle=\langle|\phi|\rangle$. In order to define the equation of state, we first define a shifted field $\tilde{\sigma}=\sigma-\langle\sigma\rangle=\sigma-M$. Then the equation of state is simply the relation

$$
\langle\tilde{\sigma}\rangle=0
$$

This relation has been expanded to order $\epsilon^{2}$ by Brézin, Wallace and Wilson [9]. The result can be expressed conveniently in terms of the variables $y \equiv H / M^{\delta}$ and $x \equiv t / M^{1 / \beta}$ as

$$
y=f(x)
$$

where the function $f(x)$ was calculated to order $\epsilon^{2}$ in [9], and is given in the appendix. The units in which $H$ and $M$ are measured are chosen so that $f(0)=1$ corresponds to $t=0$ and $f(-1)=0$ corresponds to the $t<0, H=0$ coexistence curve. Knowing $f(x)$, we can calculate the value of the order parameter $M$ for a given $H$ and $t$ using (3.4) . The behaviour of the order parameter is illustrated in Figure 1. This figure and the other ones in this section should be viewed as illustrations of qualitative behaviour rather than quantitative predictions because 
they are based on setting $\epsilon=1$ in the $O\left(\epsilon^{2}\right)$ expression for $f(x)$. The values for the critical exponents themselves which we quoted in the previous section are quantitative predictions, complete with error estimates, because they are based on the much more elaborate analysis of Baker et al. [7]

From the equation of state, we can deduce the behaviour of $m_{\pi}$ and $m_{\sigma}$ at non-zero (but small) $t$ and $H$. The masses are given by

$$
m_{\sigma}^{2}=\frac{\partial H}{\partial M}
$$

and

$$
m_{\pi}^{2}=\frac{H}{M}
$$

The first relation follows directly from the definition (3.1), and the second follows from (3.2) and from assuming that $\vec{M} \| \vec{H}$, so that a small change $\delta H \perp H$ gives a small change $\delta M \perp M$ with $\delta M / \delta H=M / H$. Using the equation of state, we can rewrite (3.5) and (3.6) as

$$
m_{\pi}^{2}=M^{\delta-1} f(x)
$$

and

$$
m_{\sigma}^{2}=M^{\delta-1}\left(\delta f(x)-\frac{x}{\beta} f^{\prime}(x)\right)
$$

Hence, as suggested in [8], $\delta$ can be determined by measuring the ratio $m_{\sigma}^{2} / m_{\pi}^{2}$ at $t=0$. In general, from $f(x)$ we can find the pion and sigma masses for any $t$ and $H$.

There are two interesting limits which we will consider explicitly. First, for $t>0$ and $H \rightarrow 0$ which corresponds to $x \rightarrow \infty$, we should find the full $O(4)$ symmetry, and hence should find that the pion and sigma masses are identical. For $x \rightarrow \infty$, the function $f(x)$ from the appendix behaves as $f(x)=c x^{\gamma}$. Here, 
the constant $c$ and the exponent $\gamma$ are given to $O(\epsilon)$ and $O\left(\epsilon^{2}\right)$ respectively in (A9) and (A10) . Applying (3.7) and (3.8), we find that

$$
m_{\sigma}^{2}=m_{\pi}^{2}=c t^{\gamma} \text { for } x \rightarrow \infty
$$

consistent with the symmetry.

We can also consider the limiting case of approaching the coexistence curve. This means taking $t<0$ and $H \rightarrow 0$, which implies $x \rightarrow-1$. In this limit, $M$ tends to a nonzero constant, and so from (3.6), we obtain $m_{\pi}^{2} \propto H$, a familiar result for Goldstone bosons. The behaviour of the pion mass is illustrated in Figure 2a. The result (3.6) may look peculiar to a particle physicist who is more familiar with the zero temperature result

$$
m_{\pi}^{2}=\frac{2 m_{q}\langle\bar{q} q\rangle}{f_{\pi}^{2}} .
$$

Before considering the sigma mass, we therefore pause here to explain how (3.10) and (3.6) are related. We have seen that $m_{q} \sim H$ and that the order parameter $\langle\bar{q} q\rangle \sim\langle\sigma\rangle \sim M$. At zero temperature, $f_{\pi}$ is defined in terms of the axial current by the relation

$$
\left\langle 0\left|A_{\mu}^{\alpha}(0)\right| \pi^{\beta}(q)\right\rangle=i f_{\pi} q_{\mu} \delta^{\alpha \beta}
$$

In the zero temperature linear sigma model, the axial current is given by

$$
A_{\mu}^{\alpha}(x)=\sigma(x) \partial_{\mu} \pi^{\alpha}(x)-\pi^{\alpha}(x) \partial_{\mu} \sigma(x)
$$

which means that $f_{\pi}$ defined in (3.10) is simply

$$
f_{\pi}=\langle 0|\sigma| 0\rangle
$$

This result suggests that we make the identification $f_{\pi} \sim M$, which does indeed make (3.6) and (3.10) equivalent. However, it is important to remember that using 
the linear sigma model at zero temperature can not be justified by a universality argument in the way that using it near $T=T_{c}$ can. Hence the argument of this paragraph is not a derivation of (3.6) from the zero temperature result (3.10) . (3.6) is valid near $T=T_{c}$ while (3.10) is valid at $T=0$. Also, $m_{\pi}$ in (3.10) is a mass in a $3+1$ dimensional Lorentz invariant theory, while $m_{\pi}$ in (3.6) is an inverse susceptibility in a 3 dimensional theory. We have simply shown that a reader familiar with one expression should not be surprised by the other.

The behaviour of the sigma mass at the coexistence curve is trickier to obtain than that of the pion mass. First, we note that in mean field theory $(\epsilon=0)$ the equation of state is simply $y=f(x)=1+x$, and $m_{\sigma}$ is easily evaluated using (3.8) . For $H \rightarrow 0$ at fixed $t<0$ the result is

$$
m_{\sigma}^{2}=\left(\frac{\delta}{|t|^{\beta}}\right) H+\frac{|t|^{\beta(\delta-1)}}{\beta} .
$$

Hence, in mean field theory $m_{\sigma}^{2}$ decreases with $H$ to a non-zero value at $H=0$. However, for $d<4$ when fluctuations are important, the result is quite different. In words, fluctuations of the massless pions produce new infrared singularities in the longitudinal susceptibility, or, equivalently, make the sigma massless. Now, let us see how this result can be obtained from the equation of state $[10,11]$. In the limit $H \rightarrow 0, f(x) \sim H$ while $f^{\prime}(x)$, we will see, tends to zero more slowly. Hence, the second term in (3.8) is dominant and gives

$$
\frac{\beta m_{\sigma}^{2}}{M^{\delta-1}} \rightarrow f^{\prime}(x) \text { for } x \rightarrow-1 \text {. }
$$

The difficulty is that from the expression (A11) for $f(x)$ valid for $x \rightarrow-1$, we notice that $f^{\prime}(x)$ contains divergent terms like $\epsilon \log (x+1), \epsilon^{2} \log ^{2}(x+1)$ and $\epsilon^{2} \log (x+1)$. These terms do not exponentiate to $f^{\prime}(x) \sim(x+1)^{p}$, contrary to the claims of [8]. After some algebra [11], one finds the result

$$
\left(\frac{\beta m_{\sigma}^{2}}{M^{\delta-1}}\right)^{-1} \rightarrow c_{1}+c_{2} y^{-\epsilon / 2}
$$

Both the terms on the right side of (3.16) must be kept because they differ in their 
exponents only by order $\epsilon$. Also for this reason, the constants $c_{1}$ and $c_{2}$ given in the appendix are known only to order $\epsilon$ even though $f(x)$ is known to order $\epsilon^{2}$.

Qualitatively, as $H$ is lowered at fixed $t<0$, at first the $c_{1}$ term dominates and $m_{\sigma}^{2}$ appears to be decreasing toward a non-zero value at $H=0$ as in the mean field result. Then, the $c_{2}$ term takes over and one finds that in fact the sigma mass goes to zero like $m_{\sigma}^{2} \propto H^{\epsilon / 2}$. The behaviour of the sigma meson mass is illustrated in Figures $2 \mathrm{~b}$ and 3. In future lattice simulations, as $m_{q}$ is lowered toward zero, this behaviour should be observed. This result is an example of the power of the renormalization group techniques in obtaining universal results. If we had chosen a specific model, say that of Gocksch [6], or the Nambu-Jona-Lasinio model of Hatsuda and Kunihiro [12], we would have been able to calculate non-universal quantities far from $T_{c}$, but would basically have been limited to using mean field theory, as those authors do. Then, we would have reached the incorrect conclusion that the sigma has a non-zero mass in the chiral limit below $T_{c}$. Here, by restricting ourselves to calculating universal quantities, we are limited to the region near the critical point, but we can be confident in our results regardless of which specific model is correct and can include the effect of fluctuations.

\section{4. $\rho$ and $a 1$ Correlation Functions}

To this point, we have discussed the correlation functions in the pion and sigma channels only. It is certainly possible to construct other spatial correlation functions. The next-simplest are those associated with the $\rho$ (Lorentz vector, isospin vector) and $A_{1}$ (Lorentz vector, isospin axial vector) mesons. In Gocksch's model [6], these have correlation lengths given simply by $2 m_{Q}(T)$ where $m_{Q}=\pi T+g\langle\sigma\rangle$ is the mass of the constituent quarks in his model. Since these constituent quarks are fermions, they have a Matsubara mass of $\pi T . g$ is a coupling constant. In any model, there is bound to be a model dependent contribution to these correlation lengths which is smooth at $T_{c}$. However, the pions and sigma also contribute to the $\rho$ and $A_{1}$ correlation lengths since operators with the appropriate symmetries 
can be constructed from the pion and sigma operators. In particular,

$$
\rho_{\alpha}^{i}=\epsilon_{\alpha \beta \gamma} \pi_{\beta} \nabla^{i} \pi_{\gamma}
$$

and

$$
\left(A_{1}\right)_{\alpha}^{i}=\sigma \nabla^{i} \pi_{\alpha}-\pi_{\alpha} \nabla^{i} \sigma
$$

Hence, the pions and sigma can make a universal non-analytic contribution to the $\rho$ and $A_{1}$ correlation lengths at the critical point. In this section, we calculate this contribution for $t>0$ and $H=0$.

Although we will try to define all the quantities we use, for those interested in further details we note that we are following the notation and conventions of Amit [3]. In the region $t>0$ and $H=0$ the symmetry is unbroken and hence there is no distinction between the $\sigma$ and $\pi$ operators which we will call $\phi^{\alpha}$ and the $\rho$ and $A_{1}$ operators which we combine into $\mathcal{O}_{i}^{\alpha \beta} \equiv \epsilon^{\alpha \beta \gamma \delta} \phi^{\gamma} \nabla_{i} \phi^{\delta}$. We are interested in the scaling behaviour of the correlation function $\langle\mathcal{O}(x) \mathcal{O}(0)\rangle \equiv \Gamma^{(0,0,2)}$. In general, by $\Gamma^{(m, n, p)}$ we mean the $m$-point vertex function with $n$ insertions of $\phi^{2}$ and $p$ insertions of $\mathcal{O}$. The scaling behaviour of vertex functions involving a composite operator like $\mathcal{O}$ is determined by $\eta, \nu$, and the anomalous dimension of the operator. Here we are fortunate because the operators $\mathcal{O}_{i}$ are the "conserved" currents of the chiral symmetry, where in the 3 dimensional theory this means $\nabla^{i} \mathcal{O}_{i}=0$. The Ward identities arising from chiral symmetry imply that the $\mathcal{O}$ are not subject to renormalization [13], or, equivalently, that their anomalous dimensions are zero.

For $H=0$, the vertex function $\Gamma^{(0,0,2)}$ is a function of the external momentum $k$, the reduced temperature $t$, the renormalization point $\kappa$, and the $\phi^{4}$ coupling constant $\lambda$. At the fixed point of the renormalization group, $\lambda$ is a constant and the vertex function satisfies the renormalization group equation [3]

$$
\left\{\kappa \frac{\partial}{\partial \kappa}-\left(\frac{1}{\nu}-2\right) t \frac{\partial}{\partial t}\right\} \Gamma^{(0,0,2)}(k, t, \kappa)=B .
$$

The right hand side of the equation is non-zero because although $\Gamma^{(0,0,2)}$ is not 
subject to multiplicative renormalization, it is additively renormalized. However, $B$ does not depend on either $k$ or $t$ [3], and therefore does not contribute to the non-analytic behaviour at the critical point. Therefore, in what follows we will drop $B$. As a consequence of (4.3), the vertex function has the form

$$
\Gamma^{(0,0,2)}(k, t, \kappa)=F\left(\kappa^{1 / \theta}, k\right)
$$

where $\theta=\frac{1}{\nu}-2$. By dimensional analysis, we know that

$$
\Gamma^{(0,0,2)}(k, t, \kappa)=b^{d-2} \Gamma^{(0,0,2)}\left(\frac{k}{b}, \frac{t}{b^{2}}, \frac{\kappa}{b}\right) .
$$

This means that (4.4) becomes

$$
\Gamma^{(0,0,2)}=b^{d-2} F\left(\frac{\kappa}{b}\left(\frac{t}{b^{2}}\right)^{1 / \theta}, \frac{k}{b}\right) .
$$

To this point, $b$ has been arbitrary. Therefore, we can choose it strategically. With the choice $b=\kappa\left(t / \kappa^{2}\right)^{\nu}$, we find

$$
\Gamma^{(0,0,2)} \sim t^{\nu(d-2)} g\left(k t^{-\nu}\right)
$$

where the function $g(x)$ satisfies $g(0)=$ constant. If, on the other hand, we choose $b=k$, the result is

$$
\Gamma^{(0,0,2)} \sim k^{d-2} \tilde{g}\left(k t^{-\nu}\right)
$$

where the function $\tilde{g}(x)$ tends to a constant for $x \rightarrow \infty$. (4.7), which gives the behaviour of $\Gamma^{(0,0,2)}$ as a function of $t$ for $k=0$, and (4.8), which gives the behaviour for $t=0$ as a function of $k$, describe the non-analytic part of the correlation function. As we mentioned earlier, there will also be a model dependent but analytic mass term for the $\rho$ and $A_{1}$. Except near the critical point, this smooth term is presumably larger than the non-analytic term whose effects we have calculated. In order to observe (4.7) and (4.8), future lattice simulations will have to get close enough to the critical point that the non-analytic term dominates the analytic term. 


\section{The Influence of the Strange Quark}

To this point in this paper, we have described a world with two massless quarks, and hence we have implicitly been taking the strange quark mass to be infinite. If the strange quark is massless, then Pisarski and Wilczek showed [1] that the chiral phase transition is first order. Hence, as the strange quark mass is reduced from infinite to zero, at some point the phase transition must change from second order to first order. This point is called a tricritical point. There is numerical evidence [14] that when the strange quark has its physical mass, the transition is second order. Hence, we devote most of this paper to analyzing the second order phase transition. However, in a lattice simulation, the strange quark mass could be tuned to just the right value to reach the tricritical point. In this section, we discuss the critical exponents that would be observed in such a simulation.

Let us consider the effect of adding a massive but not infinitely massive strange quark to the two flavor theory. This will not introduce any new fields which become massless at $T_{c}$, and so the arguments leading to the free energy (2.4) are still valid. The only effect of the strange quark, then, is to renormalize the couplings. Renormalizing $\mu^{2}$ simply shifts $T_{c}$, as does renormalizing $\lambda$ unless $\lambda$ becomes negative. In that case, one can no longer truncate the Landau-Ginzburg free energy at fourth order. After adding a sixth order term, the free energy becomes

$$
F=\int d^{3} x\left\{\frac{1}{2}(\nabla \phi)^{2}+\frac{\mu^{2}}{2} \phi^{2}+\frac{\lambda}{4}\left(\phi^{2}\right)^{2}+\frac{\kappa}{6}\left(\phi^{2}\right)^{3}-H \sigma\right\}
$$

While for positive $\lambda, \phi^{2}$ increases continuously from zero as $\mu^{2}$ goes through zero,

for negative $\lambda, \phi^{2}$ jumps discontinuously from zero to $|\lambda| /(2 \kappa)$ when $\mu^{2}$ goes through $\lambda^{2} /(4 \kappa)$. Hence, the phase transition has become first order. Thus at the value of $m_{s}$ where $\lambda=0$, the phase transition changes continuously from second order to first order.

The singularities of thermodynamic functions near tricritical points, like the singularities near ordinary critical points, are universal. Hence, it is natural to 
propose [1] that QCD with two massless flavors of quarks and with $T$ near $T_{c}$ and $m_{s}$ near its tricritical value is in the universality class of the $\phi^{6}$ Landau-Ginzburg model (5.1) . This model has been studied extensively [15]. Because the $\phi^{6}$ interaction is strictly renormalizable in three dimensions, this model is much simpler to analyze than the $\phi^{4}$ model of the ordinary critical point. No $\epsilon$ expansion is necessary, and the critical exponents all take their mean field values. There are calculable logarithmic corrections to the scaling behaviour of thermodynamic functions [15], but we will limit ourselves here to determining the mean field tricritical exponents.

In mean field theory, the correlation function in momentum space is simply $G_{\alpha \beta}(k)=\delta_{\alpha \beta}\left(k^{2}+\mu^{2}\right)^{-1}$. Since $\mu^{2} \sim t$, this gives the exponents $\eta=0, \gamma=1$ and $\nu=1 / 2$. To calculate $\alpha$ and $\beta$, we minimize $F$ for $H=\lambda=\nabla \phi=0$, and find $\alpha=1 / 2$ and $\beta=1 / 4$. To calculate $\delta$, we minimize $F$ for $t=\lambda=\nabla \phi=0$ and find $\delta=5$.

The result for the specific heat exponent $\alpha$ is particularly interesting, since it means that the specific heat diverges at the tricritical point, unlike at the ordinary critical point. This means that whereas for $m_{s}$ large enough that the transition is second order the specific heat $C(T)$ has a cusp but is finite at $T=T_{c}$, as $m_{s}$ is lowered to the tricritical value $C\left(T_{c}\right)$ should increase since at the tricritical point it diverges. This behaviour should be seen in future lattice simulations.

Finally, at a tricritical point there is one more relevant operator than at a critical point, since two physical quantities $\left(t\right.$ and $\left.m_{s}\right)$ must be tuned to reach a tricritical point. Hence, a new exponent $\phi_{t}$, the crossover exponent, is required. For $\lambda \neq 0$, tricritical behaviour will be seen only for $|t|>t^{*}$, while for $|t|<t^{*}$, either ordinary critical behaviour or first order behaviour (depending on the sign of $\lambda$ ) results. $t^{*}$ depends on $\lambda$ according to

$$
t^{*} \sim \lambda^{1 / \phi_{t}}
$$

The mean field value of $\phi_{t}$ is obtained by minimizing the free energy $F$ for $H=$ 
$\nabla \phi=0$, and is $\phi_{t}=1 / 2$. These mean field tricritical exponents, $\alpha=1 / 2, \beta=1 / 4$, $\gamma=1, \delta=5, \eta=0, \nu=1 / 2$, and $\phi_{t}=1 / 2$ would describe the real world if $m_{s}$ were smaller than it is, and will describe future lattice simulations with $m_{s}$ chosen appropriately.

\section{Dynamic Critical Phenomena}

To this point, we have discussed the static critical phenomena appropriate to the equilibrium properties of the QCD plasma near its phase transition. To discuss dynamical phenomena, we need equations of motion. In zero temperature scalar field theory, Lorentz invariance requires that these equations have no first order time derivatives. In the finite temperature theory appropriate for discussing critical behaviour, however, there is no Lorentz invariance, and hence we should expect first order time derivatives. In analogy with the notion of static universality, one finds that the dynamics of the long wavelength modes groups theories into dynamic universality classes containing theories described by the same equations of motion. To specify the dynamic universality class, one needs to specify more than the dimension of space and the number of components of the order parameter. Hence, there are usually several different dynamic universality classes which are all in the same static universality class. In particular, it is necessary to specify whether or not the order parameter is conserved, and which other quantities are conserved. Thus a ferromagnet will have very different dynamical behaviour from an antiferromagnet with the same number of components, even though the static universality class is the same. The ferromagnet will have a much more difficult time thermalizing long-wavelength fluctuations, since in the $k \rightarrow 0$ limit they are rigorously stable.

From this point of view, two flavor QCD behaves as an antiferromagnet. Its order parameter, the expectation value of a scalar or pseudoscalar quark bilinear, is not a conserved quantity. (It is quantities of the form $\bar{q} \gamma_{0} q$, not $\bar{q} q$, that are conserved). This means that a model similar to model $\mathrm{G}$ of Halperin and Hohenberg 
[16], is appropriate. Model $\mathrm{G}$ is formulated for a three component order parameter. We show below how to formulate it for the $N=4$ order parameter appropriate for QCD. One main result of the theory concerns the rate of critical slowing down near the transition. Just as the correlation length in space diverges at the critical point, so does the correlation time for dynamics. Its scaling property is conventionally written in terms of a critical exponent $z$, such that the correlation time scales as $\xi^{z}$, where $\xi$ is the correlation length. For the model in question, we show below that the exponent $z$ governing critical slowing down is predicted to be $d / 2=3 / 2$.

Let us now consider how model G of [16] must be modified to deal with the four component order parameter of interest. As we discussed above, it is important to find the conserved quantities. First, there is the energy. However, it is shown in [17] that if $\alpha$, the specific heat exponent, is negative then the dynamics of the order parameter is not affected by the presence of a conserved energy. However, the six generators of $O(4)$ rotations are associated with six conserved quantities which can be written as an antisymmetric tensor

$$
J_{\alpha \beta}=\int d^{3} x j_{\alpha \beta}
$$

At the level of the unrenormalized Lagrangian, $j_{\alpha}$ can be expressed in terms of the order parameter as

$$
j_{\alpha \beta}=\epsilon_{\alpha \beta \gamma \delta} \phi_{\alpha} \frac{\partial}{\partial t} \phi_{\beta}
$$

but this relation need not hold upon renormalization. (Note that since we are interested in the critical phenomena, by renormalization we mean renormalization towards the infrared.) In model $\mathrm{G}$, the symmetry is $O(3)$, so there are 3 conserved quantities instead of 6 . The 3 conserved quantities are the magnetization, while the nonconserved order parameter is the staggered magnetization of the antiferromagnet. The equations of motion for $j$ and $\phi$ contain two types of terms. There are dissipative terms which damp the system toward the equilibrium configuration and so called mode-mode coupling terms. The latter reflect the Poisson bracket 
relations among the fields. In our case, these are

$$
\left[\phi_{\alpha}, J_{\beta \gamma}\right]=\epsilon_{\alpha \beta \gamma \delta} \phi_{\delta}
$$

and

$$
\left[J_{\alpha \beta}, J_{\gamma \delta}\right]=\delta_{\beta \gamma} J_{\alpha \delta}-\delta_{\alpha \gamma} J_{\beta \delta}-\delta_{\beta \delta} J_{\alpha \gamma}+\delta_{\alpha \delta} J_{\beta \gamma}
$$

The equations of motion are

$$
\frac{\partial \phi_{\alpha}}{\partial t}=-\Gamma \frac{\delta F}{\delta \phi_{\alpha}}+g\left[\phi_{\alpha}, j_{\beta \gamma}\right] \frac{\delta F}{\delta j_{\beta \gamma}}+\theta
$$

and

$$
\frac{\partial j_{\alpha \beta}}{\partial t}=\gamma \nabla^{2} \frac{\delta F}{\delta j_{\alpha \beta}}+g\left[j_{\alpha \beta}, \phi_{\gamma}\right] \frac{\delta F}{\delta \phi_{\gamma}}+g\left[j_{\alpha \beta}, j_{\gamma \delta}\right] \frac{\delta F}{\delta j_{\gamma \delta}}+\zeta,
$$

where the free energy $F$ is given by

$$
F=\int d^{3} x\left\{\frac{1}{2} \partial^{i} \phi^{\alpha} \partial_{i} \phi_{\alpha}+\frac{\mu^{2}}{2} \phi^{\alpha} \phi_{\alpha}+\frac{\lambda}{4}\left(\phi^{\alpha} \phi_{\alpha}\right)^{2}+\frac{1}{2 \chi} j_{\alpha \beta} j^{\alpha \beta}-H \sigma\right\}
$$

$\theta$ and $\zeta$ are Langevin noise terms. The difference between the dissipative terms in the two equations of motion reflects the fact that $j$ is conserved, and hence a spatially constant $j$ can not dissipate. Note that $j$ appears in the free energy only as $j^{2}$. This is because any higher order terms like $j^{2} \phi^{2}$ or those involving $\nabla j$ are irrelevant. Because of the form of $F$, it turns out that the term in the equation of motion (6.6) involving (6.4) is zero. The equations of motion given above determine the universal dynamics of the long wavelength modes of interest near the critical point.

With equations of motion in hand, Halperin et al. [16] go on to formulate dynamic renormalization group transformations. These are obtained by starting 
with the theory defined with an ultraviolet cut-off $\Lambda$, integrating out modes in the momentum shell between $b^{-1} \Lambda$ and $\Lambda$ and then rescaling according to

$$
\begin{aligned}
x \rightarrow x^{\prime} & =b^{-1} x \\
\phi \rightarrow \phi^{\prime} & =b^{(d-2+\eta) / 2} \phi \\
j \rightarrow j^{\prime} & =b^{c} j \\
t \rightarrow t^{\prime} & =b^{-z} t .
\end{aligned}
$$

After one such transformation, one obtains a new free energy and new equations of motion. If one works in the $\epsilon$ expansion, these are related to the former $F$ and equations of motion simply by a transformation of the parameters $\mu, \lambda, \Gamma, \gamma, g$, and $\chi$. Repeated application of the renormalization group transformation therefore leads to recursion relations for these parameters. The next step is to find the fixed point of the transformation. Just as in the static case the fixed point condition fixes $\eta$, here it fixes $\eta, c$, and $z$. Fortunately, to obtain $c$ and $z$ we only need the particularly simple recursion relations for $\chi$ and $g$. Because $j$ only appears quadratically in the free energy, the recursion relation for $\chi$ is

$$
\chi^{-1} \rightarrow b^{d-2 c} \chi^{-1}
$$

and this is valid to all orders in $\epsilon$. The terms in the equations of motion proportional to $g$ are consequences of the $O(4)$ symmetry, and as a result the Ward identities enforce the recursion relation

$$
g \rightarrow b^{z-d+c} g
$$

to all orders in $\epsilon$. From (6.9) and (6.10) it is clear that at a fixed point one must have $c=d / 2$ and, as advertised earlier, $z=d / 2$. 


\section{Implications on the Lattice and in the Real World}

Finite temperature lattice QCD simulations are ideally suited to testing many of the predictions made in this paper. The static correlation functions of the three dimensional theory are natural objects to consider in finite temperature (Euclidean) simulations. Also, it is much easier to vary parameters like the temperature and the bare quark mass in a lattice simulation than in a real experiment. Hence, it should be possible to measure the static critical exponents of section 2 , the equation of state and the scaling behaviour of the pion and sigma masses of section 3 , the behaviour of the $\rho$ and $A_{1}$ correlation functions of section 4 , and the static tricritical exponents of section 5 .

Present simulations [14,18] provide strong evidence that the QCD phase transition is second order, and that the order parameter is $\mathcal{M}[1]$. However, there are several reasons why present simulations can not yet be used to test the more detailed predictions of this paper. The fundamental reason is that in all simulations to date, the bare quark mass has been so large that correlation lengths do not get very long at $T_{c}$. For example, in the work of Bernard et al. [18], the correlation length in the pion channel at $T_{c}$ is only about 2.5 lattice lengths. Hence, in order to study the behaviour nearer to the critical point and to measure universal properties, we must wait for simulations with smaller quark masses. The fundamental problem is that long correlation lengths are necessarily accompanied by numerical critical slowing down, and this makes simulations challenging.

Another hurdle to be overcome before lattice simulations can measure the critical properties of the QCD phase transition is that any lattice implementation of fermion fields only exhibits the full chiral symmetry in the continuum limit. If Wilson fermions are used, there is no chiral symmetry at all on the lattice. If Kogut-Susskind fermions are used, four flavors of fermions are required, but there is a continuous $U(1) \times U(1)$ chiral symmetry on the lattice. This should give a phase transition in the universality class of the $N=2$ magnet, and has been discussed in [19]. In order to study two flavors of fermions, one takes the square root of the 
fermion determinant in the lattice action. It is not at all clear what this does to the lattice chiral symmetries. Hence, for Kogut-Susskind as for Wilson fermions, before we are able to test our predictions for the critical phenomena we need finer lattices so that extrapolation to the continuum limit can be done. A necessary condition for doing this is that the results for Kogut-Susskind and Wilson fermions agree when so extrapolated. This condition has not yet been met [20].

Hence, while we cannot test our detailed predictions against current lattice simulations, we are confident that in the future with finer lattices and longer correlation lengths, simulations will be able to measure critical exponents, correlation functions, and the equation of state, and verify our results.

Let us now turn to real experiments, as opposed to those on the lattice. Here, we are not free to dial the bare quark mass. We will see that this is unfortunate, particularly in our discussion of heavy ion collisions. First, however, let us dispose of two other possible arenas for testing our results. The QCD phase transition certainly occurred in the early universe. Indeed, much work has been done on possible observable effects of this transition, if it is first order. Unfortunately, for physical values of the quark masses, we have seen that the transition is second order. We can think of no observable consequences of a second order QCD phase transition in the early universe. In [1], it was noted that since certain antiferromagnets including dysprosium have order parameters in the $N=4$ universality class [21], experiments on the phase transition in these materials would help us understand the QCD transition. Alas, in these magnets there is a quartic operator like $\left(\pi_{1}^{2}+\right.$ $\left.\pi_{2}^{2}\right)\left(\pi_{3}^{2}+\sigma^{2}\right)$ which is allowed by the microscopic hamiltonian of the magnets [22], but not by that of QCD. This operator makes the symmetric Heisenberg fixed point infrared unstable, and either makes the phase transition first order or makes it second order but governed by an anisotropic fixed point. Hence, neither cosmology nor dysprosium are suitable arenas for learning about the QCD phase transition.

Now, we turn to relativistic heavy ion collisions. In Bjorken's [23] picture of such a collision, a volume of hot plasma forms and quickly reaches thermal 
equilibrium. In the center of mass frame, the incident nuclei are both Lorentz contracted into pancake shapes. They pass through each other, and leave behind a region of hot vacuum. In translation, this means that the baryon number of the incident nuclei ends up in that part of the plasma heading approximately down the beam pipes, and the central rapidity region consists of plasma with approximately zero baryon number. In the remainder of this paper, we will attempt to use what we have learned about the critical phenomena associated with the chiral phase transition to study the behaviour of the plasma in the central rapidity region as it expands and cools through $T=T_{c}$ and eventually hadronizes and becomes pions which fly off and are detected.

The defining characteristic of a second order phase transition is the divergence of correlation lengths. How could this feature be observed here? Large volumes of space with the order parameter correlated and pointing in a direction different than the true vacuum (i.e. sigma) direction will become regions in which the order parameter oscillates coherently about the sigma direction. After hadronization, correlated volumes will turn into regions of space where the ratio of the number of charged pions to neutral pions has some fixed value. Since, in the standard scenario of Bjorken [23], different positions in the plasma along the beam direction become different longitudinal momenta (actually, different rapidities) as the plasma expands, one would hope that a signal of a second order phase transition would be fluctuations in the ratio of charged to neutral pions as a function of rapidity. Coherent evolution of a classical pion field has been considered before by Anselm and Ryskin [24] and by Blaizot and Krzywicki [25]. However, these authors considered neither an equilibrium second order phase transition as we do in this section, nor a quench as we will in the next section.

A prerequisite for the fluctuations discussed above to be observable is that the correlation length must get long compared, say, to $T_{c}$. To determine whether this does indeed happen, we must leave our universality safety net behind, since neither $T_{c}$ nor the magnitudes of correlation lengths are universal. From Figure 2a, it is immediately obvious that we have a problem. The longest correlation length 
is in the pion channel, and the pion mass is increasing with temperature. This suggests that the pion mass at $T_{c}$ is larger than $m_{\pi}(T=0)=135 \mathrm{MeV}$. This is consistent with the fact that in many models the pion mass increases from its zero temperature value as the temperature is increased from zero. This result has been obtained in chiral perturbation theory using the nonlinear sigma model [26] and also for the linear sigma model [27]. Hence, it seems clear that the longest correlation length at $T_{c}$ will be shorter than $(135 \mathrm{MeV})^{-1}$. This is to be compared to $T_{c}$ itself, which for the case of two massless quarks is around $140 \mathrm{MeV}$ [18]. Hence, even though the quark masses are indeed small $(\sim 10 \mathrm{MeV})$, the magnetic field $H$ proportional to $m_{q}$ is large enough to prevent any correlation lengths from reaching interesting values.

There is an appropriate quantitative criterion to determine whether a near equilibrium second order phase transition leads to dramatic effects. One compares the energy in a correlation volume just below $T_{c}$ with the zero temperature pion mass to determine whether or not the correlated volume can become a large number of pions. Using current lattice simulations [18], we can make a crude attempt at this comparison. The sum of the energy and pressure in a correlation volume is about $1 / 4$ the zero temperature $\rho$ mass. Taken literally, this means that each correlation volume becomes only one or two pions in the detector. As we have mentioned, current simulations are subject to many caveats, and so this estimate should not be taken literally. Nevertheless, it seems clear that the physical value of $m_{q}$ is large enough that in an equilibrium phase transition a correlation volume at $T_{c}$ does not evolve into a large number of correlated zero temperature pions. This is not encouraging.

We pause here for an aside. The reader may be wondering why, when the seemingly small equal quark mass $m_{u}=m_{d}=m_{q}$ has such deleterious effects, we have completely neglected the difference between the up and down quark masses. It was noted in [1] that unequal quark masses allow terms of the type

$$
\Delta F \propto(\delta m)^{2}\left(\sigma^{2}-\pi_{3}^{2}+\pi_{1}^{2}+\pi_{2}^{2}\right)
$$


If one is close enough to the critical point that this term matters, one will discover an anisotropic fixed point rather than the symmetric Heisenberg fixed point. However, while the effect of a common quark mass, namely the mass of the pion, is comparable to $T_{c}$, the effect of (7.1) is much smaller. For example, the QCD contribution to the difference in mass between the charged and neutral kaons is about $5 \mathrm{MeV}$ [28]. Therefore, we need not worry about (7.1) in real experiments since it is much less important than the effect of the "magnetic field" proportional to the common up and down quark mass. Of course, (7.1) could be introduced and studied on the lattice.

It seems clear that if the standard scenario for heavy ion collisions in which the QCD plasma cools through $T_{c}$ while staying close to thermal equilibrium is correct, then no correlation lengths will get long enough for there to be any dramatic observable effects of the phase transition. The chiral "transition", like the confinement/deconfinement "transition," will be a smooth crossover. If we were able to dial down the quark masses and hence the pion mass, phenomena associated with a second order transition would become more prominent. Alas, in the real world, unlike on a lattice, we have no such freedom.

\section{Non-equilibrium Phenomena in Relativistic Heavy Ion Collisions}

We are not done yet. The gloomy paragraph with which we ended the preceding section began with a conditional sentence. In this section we will consider the observable effects in heavy ion collisions if the plasma does not stay close to

thermal equilibrium through the transition. There are tantalizing hints in cosmic ray physics that point in this direction. Among the zoo of high energy cosmic ray events known are a particularly peculiar class of events called Centauros [29]. These are events with total energy of order $1000 \mathrm{TeV}$ in which many (of order 100) charged hadrons each with energies of a several $\mathrm{TeV}$ and very few photons or electrons are seen in a cosmic ray induced shower. In the sample of events in [29], there were 5 Centauros, representing about $1 \%$ of the events seen with energy 
of the appropriate order of magnitude. Centauros are peculiar because so many charged pions are observed without any of the gammas that would indicate the decay of neutral pions. This apparent violation of isospin invariance is puzzling, unless one thinks of it in the language of a second order phase transition in which these events can be interpreted as the creation of a volume of QCD plasma in which the $\phi$ field has fluctuated throughout most of the plasma in some direction in the $\pi_{1}-\pi_{2}$ plane. This implies correlation throughout most of a volume of plasma large enough that it becomes about 100 zero temperature pions. We convinced ourselves above that this could not happen if the plasma remains close to thermal equilibrium. Hence, these Centauro events provide a tantalizing hint that it might be wise to consider the effects of going from the symmetric phase above $T_{c}$ to the ordered phase rapidly without maintaining thermal equilibrium. This process, called quenching, has been much studied in condensed matter physics.

We noted in the previous section that if the plasma stays close to equilibrium, one finds below $T_{c}$ that the energy in a correlation volume is small compared to the zero temperature pion mass. However, since the energy density at temperatures well above $T_{c}$ is much higher than that below $T_{c}$, it is reasonable to hope that a quench, in which the energy density does not decrease in a quasi-equilibrium fashion through $T_{c}$, has a better chance of producing correlated volumes of plasma which evolve into many zero temperature pions. In this section we will begin to analyze the observable effects if relativistic heavy ion collisions proceed via quenching. In a real relativistic heavy ion collision, the phase transition will probably occur by something in between a slow equilibrium process and a quench. Only experiments can determine which description is more appropriate.

Let us begin by describing more carefully what a quench is, in the context of a Heisenberg magnet in greater than two dimensions with no applied magnetic field. The system starts at equilibrium at a temperature well above $T_{c}$, fluctuating among an ensemble of configurations with short correlation lengths. One then imagines turning the temperature instantaneously to zero. This means that the equilibrium configuration is now an ordered state with the field aligned throughout 
space. However, this is not the configuration in which the system finds itself. It is in one configuration from the ensemble appropriate to a high temperature. This configuration then evolves according to the zero temperature equations of motion (i.e. microcanonically, with no thermal fluctuations.) In a condensed matter system, the appropriate equations of motion are those of Section 6 , and in particular they are not Lorentz invariant. What is found [30] is that the size of correlated domains grows with time in such a way that after a brief initial period the correlation function has the simple scaling form

$$
C(r, t) \equiv\langle\phi(r, t) \phi(0, t)\rangle=g(r / L(t))
$$

The characteristic domain size $L$ increases with time according to $L(t) \sim t^{p}$, where the exponent $p$ depends only on $d, N$, and the dynamic universality class. It is important to note that the scaling behaviour (8.1) is obtained regardless of the initial configuration. Hence, it is not actually necessary that the initial configuration in a simulation (or in a heavy ion collision) be selected from a high temperature thermal ensemble. Any disordered initial configuration evolves to the critical behaviour (8.1) . This phenomenon is called self-organized criticality. It is also crucial for us that the domain size is not related to an equilibrium correlation length, and in particular that in an infinite system it grows without bound.

Let us now consider how the physics of quenching may be applied in relativistic heavy ion collisions. If the collision is energetic enough to create a region of plasma well above $T=T_{c}$, the $\phi$ field will indeed be fluctuating among an ensemble of disordered configurations. At the end of the process, one certainly has zero temperature. The question is what happens to the $\phi$ field in between. One idealized possibility which has been considered by many before us and which we considered in the previous section is that the system stays arbitrarily close to thermal equilibrium. Another idealization is that thermal fluctuation ceases instanteously. In a real collision, the plasma is cooling, and so cannot be exactly in thermal equilibrium. If it cools fast enough, the configuration of the $\phi$ field will "lag," and as in 
a quench the system will find itself in a configuration that is more disordered than the equilibrium configuration appropriate for the current temperature. However, it is clear that a real collision will not be an ideal quench, as thermal fluctuation will not cease instantaneously. Thus, a real collision fits in neither idealized category, but is somewhere in between. To the second idealization, the quench, we now turn.

There are several differences between a quench in the system of interest to us and in the condensed matter system we described above. First, at zero temperature we must have a Lorentz invariant field theory, with different equations of motion than those used in the condensed matter system. While the equations of motion of section 6 describe the Lorentz non-invariant dynamics of the order parameter near $T=T_{c}$, they are inappropriate for the $T=0$ dynamics of a quench. We propose to use the zero temperature linear sigma model with only pion and sigma fields. Since we will be considering energies well below the rho and nucleon masses, we need not include these degrees of freedom. The sigma could also be left out, if it were not for the fact that the appropriate initial conditions are a disordered state in which the pion and sigma fields are equivalent up to the effect of the bare quark masses. The second difference is that unlike in the magnet, the plasma in a relativistic heavy ion collision is expanding. This means that the description in terms of configurations of the field $\phi$ will not be appropriate forever. At some time, the energy density drops low enough that one has individual pions flying off towards the detectors. The third difference is that unlike in the condensed matter systems considered in [30], we must include the effects of the bare quark masses. As we saw before, these correspond to a significant magnetic field. We propose that quenching of a 4 component Heisenberg model with the three modifications we have mentioned be considered an idealized model for a heavy ion collision.

It is fortunate that the scenario we have just outlined, with the exception of the significant magnetic field, is exactly the scenario considered by Turok and Spergel [31] as a cosmological model for large scale structure formation in the early universe. They study the evolution of an $O(N)$ sigma model in an expanding universe. They find an exact scaling solution for the non-linear sigma model in the large $N$ limit, 
and do numerical simulations for the linear sigma model for $N=4$ and $N=10$. The main reason we can apply their results while we cannot use those of [30] is that Turok and Spergel use the Lorentz invariant equations of motion appropriate for our problem. They find that the size of correlated domains, $L(t)$, grows at the speed of light! We are currently [32] extending their simulations to include the effects of a magnetic field, and to vary the expansion rate. The magnetic field will qualitatively change the scenario. Instead of having correlated domains with the $\phi$ field pointing in arbitrary directions on the 3-sphere, at late times the $\phi$ field will be oscillating about the sigma direction. However, there will still be domains in which the oscillations are in different directions. If in a heavy ion collision the size of these domains grows with the speed of light as it does in our simulations [32], the phenomenological consequences for heavy ion collisions are dramatic. Of course the description we are using will only be valid for a short time. (Bjorken [23] estimates that a hydrodynamic description will be valid for about $10 \mathrm{fm} / \mathrm{c}$.) Since the plasma will be expanding slower than the speed of light, even in this short time domains which expand at the speed of light will grow to encompass large fractions of the total volume. We therefore propose that if heavy ion collisions can be modelled as a quench, this will be detected by observing clusters of pions in which all the pions in a region of rapidity are correlated in internal space. In some clusters, there will be only charged pions; in others, only neutral ones; and in all, charged and neutral pions will occur in some fixed ratio.

We can estimate the probability distribution of the ratio $R$ of the number of neutral pions to the total number of pions in a correlated region. Let us assume that the $\phi$ field in the region is initially equally likely to be pointing in any direction on the 3 -sphere. This assumption may not be strictly true because the magnetic field selects a preferred sigma direction even at high temperatures. However, we make the assumption in order to get a simple analytical result. Where $\phi$ starts will determine in which direction it ends up oscillating about the sigma direction. We define angles on the 3-sphere according to 


$$
\left(\sigma, \pi_{3}, \pi_{1}, \pi_{2}\right)=(\cos \theta, \sin \theta \cos \phi, \sin \theta \sin \phi \cos \eta, \sin \theta \sin \phi \sin \eta)
$$

Then the ratio $R$ is given by

$$
R \equiv \frac{n_{\pi^{0}}}{n_{\pi^{0}}+n_{\pi^{+} \pi^{-}}}=\frac{\sin ^{2} \theta \cos ^{2} \phi}{\sin ^{2} \theta\left(\cos ^{2} \phi+\sin ^{2} \phi\right)}=\cos ^{2} \phi
$$

Under the assumption that all initial values on the 3-sphere are equally probable, the probability distribution $\mathcal{P}(R)$ is determined by

$$
\int_{R_{1}}^{R_{2}} \mathcal{P}(R) d R=\frac{1}{\pi^{2}} \int_{0}^{2 \pi} d \eta \int_{0}^{\pi} d \theta \sin ^{2} \theta \int_{\arccos \left(\sqrt{R_{2}}\right)}^{\arccos \left(\sqrt{R_{1}}\right)} d \phi \sin \phi
$$

and turns out to be simply

$$
\mathcal{P}(R)=\frac{1}{2} R^{-1 / 2}
$$

Equivalently, the probability that $R<R_{1}$ is given by $\sqrt{R_{1}}$. If heavy ion collisions are described by a quench, there should be large regions of the collision volume containing clusters of pions in which $R$ is constant, and the values $R$ takes in different such regions should be distributed according to (8.5) .

As one application of (8.5), we note that the probability that the neutral pion fraction $R$ is less than .01 is 0.1 ! This is a graphic illustration of how different (8.5) is from what one would expect if individual pions were independently randomly distributed in isospin space. It also makes Centauro events in which less than $1 \%$ of the outgoing particles from a heavy ion collision are neutral pions seem much less surprising than they first appeared. The analysis of the Centauro data is difficult for several reasons. Most important of these is the limitation imposed by small statistics. Also, if a Centauro event occurs too high above the detector, so many secondary photons will be produced that the event will not be recognized as a Centauro. Third, in a Centauro event all of the particles from the collision strike a small region of the detector and it is impossible to isolate the central rapidity region. 
This combined with the fact that the detectors do not distinguish between charged pions and charged baryons has several unfortunate consequences. It means that events in which there are two or more correlated clusters of pions are not detected as Centauros. Only those with a single cluster are so identified. Also, the opposite type of event in which $R$ is close to 1 , will not have a dramatic signature since there will always be charged baryons present from the two initial nuclei. For all these reasons, we feel it is impossible to extract a meaningful probability distribution $\mathcal{P}(R)$ from the cosmic ray data.

When relativistic heavy ion collisions occur at high enough energies in a laboratory colliding beam facility, all of the difficulties of the cosmic ray experiments will be rapidly overcome. That will be the time to look for correlated clusters of pions,

and to look for a distribution like (8.5), and hence to determine whether these collisions proceed by a process close to the idealized quench we have considered here.

\section{Conclusions}

The future of the study of the QCD phase transition on the lattice looks promising. As simulations improve, they will begin to investigate the plethora of static critical phenomena we discussed in the first sections of this paper. Critical exponents, the equation of state, the critical behaviour of the pion and sigma susceptibilities, $\rho$ and $A_{1}$ correlation functions, and tricritical exponents are all out there waiting to be measured.

Because heavy ion collisions are dynamical processes, there are more possible scenarios and the situation is not clear cut. We have considered two idealized models. If the cooling plasma stays arbitrarily close to thermal equilibrium, our conclusions are disappointing. Because the pion is so heavy compared to $T_{c}$, correlation lengths will not become particularly long. On the other hand, in the other idealized model we considered, in which thermal fluctuations are rapidly quenched 
and the cooling plasma is in a sense maximally out of thermal equilibrium, dramatic phenomena are possible. Correlated volumes will form, and their size will not be determined by any equilibrium correlation length. Indeed they may grow at the speed of light until hadronization occurs. This will have the consequence that clusters of large numbers of pions will be detected in which the ratio of neutral to charged pions will be constant. This ratio will be different in different clusters, and will follow a probability distribution which is skewed towards having few neutral pions. We eagerly await the verdict of experiment as to which scenario is more appropriate.

Acknowledgements: We are grateful for the fruitful discussions we had with Bert Halperin. We both also acknowledge the hospitality of Harvard University, where part of this work was completed.

\section{APPENDIX}

In this appendix, we reproduce the equation of state to order $\epsilon^{2}[9]$, and give various other results used in section 3 .

The equation of state is found by doing an $\epsilon$ expansion of the relation

$$
\langle\tilde{\sigma}\rangle=0
$$

where $\tilde{\sigma}=\sigma-M$. The resulting expansion can be expressed in terms of the variables $y \equiv H / M^{\delta}$ and $x \equiv t / M^{1 / \beta}$, where

$$
\frac{1}{\beta}=2+\frac{6}{N+8} \epsilon+4 \frac{(N+5)(7-N)}{(N+8)^{3}} \epsilon^{2}+O\left(\epsilon^{3}\right)
$$

and

$$
\delta=3+\epsilon+\frac{N^{2}+14 N+60}{2(N+8)^{2}} \epsilon^{2}+O\left(\epsilon^{3}\right) .
$$

We are interested in $\epsilon=1$ and $N=4$. We choose to measure fields in units such that $y=1$ at $t=0$, and $x=-1$ at the coexistence curve $(H=0, t<0)$. The 
equation of state is

$$
y=f(x)
$$

The function $f(x)$ is given by

$$
\begin{aligned}
f(x)= & 1+x \\
+ & \frac{\epsilon}{2(N+8)}\left(1+\frac{\epsilon}{2(N+8)}[N-1+6 \ln 2-9 \ln 3+(N-1) \ln (x+1)]\right) \\
& \times(3(x+3) \ln (x+3)+(N-1)(x+1) \ln (x+1)+6 x \ln 2-9(x+1) \ln 3) \\
+ & \left(\frac{\epsilon}{2(N+8)}\right)^{2}\left\{\frac{1}{2}(10-N)(x+1)\left[\ln ^{2}(x+3)-\ln ^{2} 3\right]\right. \\
& +36\left[\ln ^{2}(x+3)-(x+1) \ln ^{2} 3+x \ln ^{2} 2\right] \\
& -54 \ln 2[\ln (x+3)+x \ln 2-(x+1) \ln 3]+3(N-1)\left(\ln \frac{27}{4}\right)(x+1) \ln (x+1) \\
& +\frac{212+17 N-4 N^{2}}{N+8}[(x+3) \ln (x+3)+2 x \ln 2-3(x+1) \ln 3] \\
& +(N-1)(x+1) \ln (x+1) \ln (x+3)-\frac{N}{2}(N-1)(x+1) \ln ^{2}(x+1) \\
& +\frac{N-1}{N+8}(19 N+92)(x+1) \ln (x+1)-2(N-1)\left[(x+6) I_{1}(\rho)-6(x+1) I_{1}(3 / 4)\right] \\
& \left.-6(N-1)\left[I_{2}(\rho)-(x+1) I_{2}(3 / 4)\right]+4(N-1)\left[I_{3}(\rho)-(x+1) I_{3}(3 / 4)\right]\right\}+O\left(\epsilon^{3}\right)
\end{aligned}
$$

where

$$
\rho \equiv \frac{x+3}{4(x+1)}
$$

and

$$
\begin{aligned}
& I_{1}(\rho) \equiv \int_{0}^{\rho} \frac{d u \ln u}{u(1-u)}[\sqrt{1-u / \rho}-1]-\int_{\rho}^{\infty} \frac{d u \ln u}{u(1-u)} \\
& I_{2}(\rho) \equiv \rho \frac{d I_{1}}{d \rho} \\
& I_{3}(\rho) \equiv I_{1}(\rho)+2 I_{2}(\rho) .
\end{aligned}
$$

The behaviour of $I_{1}(\rho)$ near the coexistence curve is given by

$$
I_{1}(\rho) \sim \frac{1}{4 \rho}\left(\ln ^{2} 4 \rho+2 \ln \rho\right), \quad \rho \rightarrow \infty .
$$


The leading terms of $f(x)$ for large $x$ are

$$
f(x) \sim\left(1+\frac{3 \ln (4 / 27)}{2(N+8)} \epsilon+O\left(\epsilon^{2}\right)\right) x^{\gamma}, \quad x \rightarrow \infty
$$

where

$$
\gamma=1+\frac{N+2}{2(N+8)} \epsilon+\frac{(N+2)\left(N^{2}+22 N+52\right)}{4(N+8)^{3}} \epsilon^{2}+O\left(\epsilon^{3}\right)
$$

Of course, this result for $\gamma$ and the results for $\beta$ and $\delta$ in (A2) and (A3) are consistent with the scaling relations (2.12) . We saw in section 3 that the behaviour (A.9) determines the sigma and pion masses for $H=0$ and $t>0$.

In section 3 , in order to determine $m_{\sigma}^{2}$ near the coexistence curve we needed the behaviour of $f^{\prime}(x)$ for $x \rightarrow-1$. In this region, $f(x)$ is given by [11]

$$
\begin{aligned}
f(x) \sim(x+1)\{1+ & \epsilon\left[\frac{N-1}{2(N+8)} \ln (x+1)+\frac{3(1+3 \ln (2 / 3))}{2(N+8)}\right] \\
+ & \epsilon^{2}\left[\frac{(N-10)(N-1)}{8(N+8)^{2}} \ln ^{2}(x+1)\right. \\
& \left.\left.+\frac{N-1}{4(N+8)^{2}}\left[N+27+18 \ln 2-9 \ln 3-\frac{60}{N+8}\right] \ln (x+1)\right]+O\left(\epsilon^{3}\right)\right\} .
\end{aligned}
$$

One next inverts (A.11) to obtain $x+1$ in terms of $y=f(x)$,

$$
(x+1)=c_{1} y+\tilde{c}_{2} y^{1-\epsilon / 2}+O\left(y^{2-O(\epsilon)}\right)
$$

differentiates the result with respect to $x$, and obtains

$$
\frac{\beta m_{\sigma}^{2}}{M^{\delta-1}} \rightarrow f^{\prime}(x)=\frac{1}{c_{1}+c_{2} y^{-\epsilon / 2}} \text { for } x \rightarrow-1
$$

with

$$
c_{1}=\frac{9}{N+8}\left(1-\frac{\epsilon}{2(N+8)}\left[(N+8) \ln 2-9 \ln 3+\frac{25 N^{2}+142 N+76}{9(N+8)}\right]\right)+O\left(\epsilon^{2}\right)
$$


and

$$
\begin{aligned}
c_{2} & =\tilde{c}_{2}\left(1-\frac{\epsilon}{2}\right) \\
& =\frac{N-1}{N+8}\left\{1-\frac{\epsilon}{2}\left(1-\frac{1}{(N+8)^{2}}[9(N+8) \ln 3+22 N+116]\right)\right\}+O\left(\epsilon^{2}\right) .
\end{aligned}
$$

As $H$ is lowered to zero at fixed $t<0, m_{\sigma}^{2}$ at first tends toward a constant, and then goes to zero according to $m_{\sigma}^{2} \propto H^{\epsilon / 2}$ when the $c_{2}$ term takes over from the $c_{1}$ term. For $N=4$, this occurs when

$$
\frac{H}{M^{\delta}} \lesssim\left(\frac{c_{2}}{c_{1}}\right)^{2 / \epsilon} \sim\left(\frac{1}{3}[1+0.96 \epsilon]\right)^{2 / \epsilon} \sim 0.4 \text { for } \epsilon=1
$$

Of course, the numerical value for $\epsilon=1$ should not be taken too seriously. The qualitative result is clear nevertheless.

We end this appendix by describing how the figures in section 3 were obtained. When evaluated at $\epsilon=1$, the expression (A.5) for $f(x)$ has several problems. First, at large $x$ it does not grow as $x^{\gamma}$. Rather, it increases like $x \ln ^{2} x$. Also, for $x \rightarrow-1$, $f(x)$ given by (A.5) does not satisfy (A.12) . In fact, for $x \lesssim-0.95, f(x)<0$ which is unphysical. Both of these problems arise because we are setting $\epsilon=1$ in an expansion of $f(x)$ to order $\epsilon^{2}$ which is valid for $\epsilon \rightarrow 0$. In order to illustrate the correct qualitative behaviour, we constructed a function $f(x)$ which smoothly interpolates between (A.9) at large $x,(\mathrm{~A} .12)$ near $x=-1$, and (A.5) in between. Using this function $f(x)$, we obtained Figure 1 by solving $H / M^{\delta}=f\left(t / M^{1 / \beta}\right)$ for $M$ at various values of $t$ and $H$. We then calculated the results for $m_{\pi}^{2}$ and $m_{\sigma}^{2}$ shown in Figures 2 and 3 using (3.6) and (3.8). Because of the limitations imposed by working at $\epsilon=1$, all three figures should be viewed as illustrations of qualitative behaviour. 


\section{REFERENCES}

1. F. Wilczek, Int. J. Mod. Phys. A7, 3911 (1992). This elaborates earlier work of R. Pisarski and F. Wilczek, Phys. Rev. D29, 338 (1984).

2. G. 't Hooft, Phys. Reports 142, 357 (1986).

3. See, for example, Modern Theory of Critical Phenomena, S.-K. Ma (Benjamin/Cummings, 1976) and Field Theory, the Renormalization Group, and Critical Phenomena, D. J. Amit (World Scientific, 1984).

4. M. Gell-Mann and M. Levy, Nuovo Cimento 16, 705 (1960).

5. P. Ginsparg, Nucl. Phys. B170, 388 (1980), and references therein.

6. A. Gocksch, Phys. Rev. Lett. 67, 1701 (1991).

7. G. Baker, B. Nickel and D. Meiron, Phys. Rev. B17, 1365 (1978), and "Compilation of 2-pt. and 4-pt. graphs for continuous spin models", unpublished, University of Guelph report (1977).

8. A. Kocić, J. B. Kogut, and M.-P. Lombardo, Illinois preprint ILL-TH-92-18, 1992.

9. E. Brézin, D. J. Wallace and K. G. Wilson, Phys. Rev. B7 232 (1973).

10. E. Brézin and D. J. Wallace, Phys. Rev. B7 1967 (1973).

11. D. J. Wallace and R. K. P. Zia, Phys. Rev. B12 5340 (1975).

12. T. Hatsuda and T. Kunihiro, Phys. Rev. Lett. 55158 (1985) and references therein.

13. Gauge Field Theories, S. Pokorski (Cambridge, 1987).

14. F. R. Brown et al., Phys. Rev. Lett. 652491 (1990).

15. I. Lawrie and S. Sarbach in Phase Transitions and Critical Phenomena 9, 1 (1984), ed. C. Domb and J. Lebowitz (Academic Press). 
16. P. C. Hohenberg and B. I. Halperin, Rev. Mod. Phys. 49, 435 (1977).

B. I. Halperin, P. C. Hohenberg, and E. D. Siggia, Phys. Rev. B13, 1299 (1976).

17. B. I. Halperin, P. C. Hohenberg, and S.-K. Ma, Phys. Rev. B10, 139 (1974).

18. C. Bernard et al., Phys. Rev. D45, 3854 (1992).

19. G. Boyd et al., Nucl. Phys. B376, 199 (1992).

20. C. Bernard et al., QCD Thermodynamics With Two Flavors of Wilson Quarks at $N_{t}=6$, Santa Barbara preprint UCSBTH-92-30.

21. P. Bak and D. Mukamel, Phys. Rev. B13, 5086 (1976).

22. Z. Barak and M. B. Walker, Phys. Rev. B25, 1969 (1982).

23. J. D. Bjorken, Phys. Rev. D27, 140 (1983).

24. A. A. Anselm and M. G. Ryskin, Phys. Lett. B266, 482 (1991).

25. J.-P. Blaizot and A. Krzywicki, Phys. Rev. D46, 246 (1992).

26. J. Gasser and H. Leutwyler, Phys. Lett. B184, 83 (1987).

27. C. Contreras and M. Loewe, Int. J. Mod. Phys. A5, 2297 (1990).

28. H. Leutwyler, Nucl. Phys. B337, 108 (1990).

29. C. M. G. Lattes, Y. Fujimoto, and S. Hasegawa, Phys. Rept. 65, 151 (1980).

30. A. J. Bray, Phys. Rev. B41, 6724 (1990); T. J. Newman, A. J. Bray, and M. A. Moore, Phys. Rev. B42, 4514 (1990); and references therein.

31. N. Turok and D. N. Spergel, Phys. Rev. Lett. 66, 3093 (1991).

32. K. Rajagopal and F. Wilczek, work in progress. 


\section{FIGURE CAPTIONS}

1) The order parameter $M$ as a function of reduced temperature $t \equiv\left(T-T_{c}\right) / T_{c}$ for magnetic fields $H=0,0.002,0.005$, and 0.02. Like $t$, both $M$ and $H$ are dimensionless. They are obtained from their dimensionful counterparts by dividing by non-universal dimensionful constants defined in such a way that for $t<0$ and $H=0$ the order parameter is given by $M=(-t)^{\beta}$, and for $t=0$ it satisfies $M=H^{1 / \delta}$.

2) $m_{\pi}^{2}$ (Figure 2a) and $m_{\sigma}^{2}$ (Figure 2b) as functions of $t$ for $H=0,0.002,0.005$, and 0.02. Since $M$ and $H$ are in dimensionless scaled units, so are $m_{\pi}^{2}$ and $m_{\sigma}^{2}$. For $t=0, m_{\pi}^{2}=H^{(\delta-1) / \delta}$ and $m_{\sigma}^{2}=\delta m_{\pi}^{2}$. For $H=0$ and $t>0$ (and for large enough $t$ for any $H$ ) $m_{\sigma}^{2}=m_{\pi}^{2} \sim t^{\gamma}$. For $t<0$ and $H \rightarrow 0, m_{\pi}^{2} \sim H$ and the sigma mass decreases to zero as shown in Figure 3.

3) $m_{\sigma}^{2}$ as a function of $H$ for $t=-0.2$. For large $H$ it behaves as if it will be non-zero at $H=0$, but in fact for $H \rightarrow 0$ it decreases like $m_{\sigma}^{2} \sim H^{p}$ where to lowest order in $\epsilon, p=\epsilon / 2=1 / 2$. 